\title{
High Extraction Efficiency Light-emitting Diodes Based on Embedded Air-gap Photonic-crystals
}

\section{Citation}

Matioli, Elison, Elizabeth Rangel, Micheal Iza, Blaise Fleury, Nathan Pfaff, James Speck, Evelyn $\mathrm{Hu}$, and Claude Weisbuch. 2010. High extraction efficiency light-emitting diodes based on embedded air-gap photonic-crystals. Applied Physics Letters 96(3): 031108.

\section{Published Version}

doi: $10.1063 / 1.3293442$

\section{Permanent link}

http://nrs.harvard.edu/urn-3:HUL.InstRepos:11005279

\section{Terms of Use}

This article was downloaded from Harvard University's DASH repository, and is made available under the terms and conditions applicable to Other Posted Material, as set forth at http:// nrs.harvard.edu/urn-3:HUL.InstRepos:dash.current.terms-of-use\#LAA

\section{Share Your Story}

The Harvard community has made this article openly available.

Please share how this access benefits you. Submit a story.

\section{Accessibility}




\title{
High extraction efficiency light-emitting diodes based on embedded air-gap photonic-crystals
}

\author{
Elison Matioli, ${ }^{1, a)}$ Elizabeth Rangel, ${ }^{1}$ Micheal Iza, ${ }^{1}$ Blaise Fleury, ${ }^{1}$ Nathan Pfaff, ${ }^{1}$ \\ James Speck, ${ }^{1}$ Evelyn Hu, ${ }^{2}$ and Claude Weisbuch ${ }^{1,3}$ \\ ${ }^{1}$ Department of Materials, University of California, Santa Barbara, California 93106-5050, USA \\ ${ }^{2}$ School of Engineering and Applied Physics, Harvard University, Cambridge, Massachusetts 02138, USA \\ ${ }^{3}$ Laboratoire de Physique de la Matière Condensée, CNRS, Ecole Polytechnique, 91128 Palaiseau, France
}

(Received 16 November 2009; accepted 21 December 2009; published online 22 January 2010)

\begin{abstract}
This letter reports on high extraction efficiency light-emitting diodes (LEDs) based on embedded two-dimensional air-gap photonic crystals (PhCs). High refractive index contrast provided by the air gaps along with high interaction of the embedded PhCs with the guided light resulted in an efficient extraction of all guided modes in the LED, in contrast to the common surface $\mathrm{PhC}$ configuration. Embedded PhC LEDs presented an enhanced directional light emission compared to non-PhC LEDs. High extraction efficiency, close to unity, provided by the encapsulated embedded PhC LEDs demonstrates the capability of this approach to achieve high efficiency devices with directional light emission. (C) 2010 American Institute of Physics. [doi:10.1063/1.3293442]
\end{abstract}

The application of photonic crystals (PhCs) as light extractors in light-emitting diodes (LEDs) has evoked increased interest over recent years. Several groups ${ }^{1-5}$ demonstrated the efficacy of PhCs in controlling the direction of light emission and enhancing the extraction efficiency of LEDs. A number of different schemes are commonly applied to increase the extraction efficiency in LEDs. While surface roughening, which is among the most widely used methods, has demonstrated extraction efficiencies as high as $80 \%$ in encapsulated LEDs, ${ }^{6}$ it offers no control over the direction of the emitted light, resulting in a Lambertian far field emission.

The most straightforward design of PhCs is on the surface of the LEDs, however the observed enhancement in light extraction, mainly in thick LED structures, is usually significantly lower than the theoretical limit, due to the limited interaction of the surface $\mathrm{PhCs}$ with some of the guided modes, notably the low order modes. ${ }^{2}$ The poor extraction of a relatively high portion of the guided light carried by these low order modes limits the enhancement on extraction efficiency by the surface $\mathrm{PhCs}$ in such structures.

The alternative approach presented in this letter is to utilize embedded air-gap PhCs within the LEDs. The greater interaction of the guided light with the embedded PhCs not only allows extraction of the low order modes but also improves the extraction of high order modes, compared to surface PhCs. ${ }^{8}$ In addition, the high refractive index contrast between $\mathrm{GaN}$ and air in these air-gap structures yields an enhanced $\mathrm{PhC}$ diffraction strength, compared to embedded dielectric-based gratings, ${ }^{9}$ and the two-dimensional (2D) nature of the $\mathrm{PhC}$ results in omnidirectional light extraction. Furthermore, the planar top surface of the embedded $\mathrm{PhC}$ LED simplifies device fabrication, and, in particular, eases issues related to making the best electrical contacts to the device. Finally, this approach mitigates possible etch-related damage to the quantum wells $(\mathrm{QWs})$, since the embedded $\mathrm{PhCs}$ are fabricated before the growth of the LED active region. These devices demonstrated close to unity extraction efficiency and highly directional light emission.

${ }^{a)}$ Electronic mail: elison.matioli@ polytechnique.org.
The schematic of the embedded PhC LED presented in this letter is shown in Fig. 1(a). Several electromagnetic guided modes are supported in such a structure, due to the fairly thick GaN slab $(\sim 4 \mu \mathrm{m})$, these are designated as low order modes, high order modes and cap-layer mode (CLM). The latter is the mode confined in the top layer above the embedded air-gap PhCs, due to their lower effective index of refraction.

A thorough optimization of the embedded PhC LED structure would require consideration of the extraction of every single supported mode in the structure; this is quite tedious due to the large number of modes. A sufficient analysis can be done by optimizing the extraction of the poorest extracted mode. In simple and general terms, all the guided modes extend spatially over the entire GaN layer, resulting in a good interaction with the embedded PhCs, except the CLM which is the only mode confined above the embedded PhCs. The CLM is not only the mode with the lowest interaction with the PhCs, but also the best excited by the QWs, justifying the importance of its optimization.

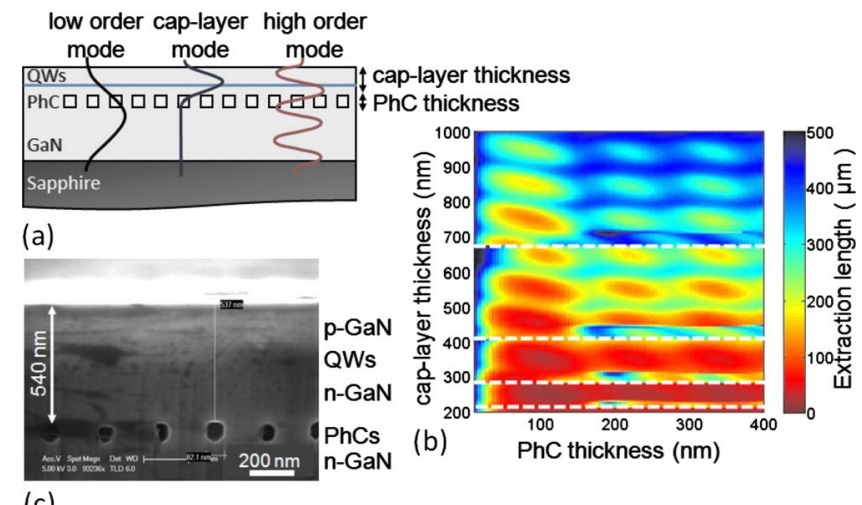

(c)

FIG. 1. (Color online) (a) Schematic of the embedded PhC LED supporting following three different families of modes: low order, high order, and CLMs. (b) Simulated extraction length of the CLM as a function of the cap-layer and embedded $\mathrm{PhC}$ thicknesses for 35\% $\mathrm{PhC}$ fill factor, $4 \mu \mathrm{m}$-thick GaN layer and $\lambda=460 \mathrm{~nm}$. (c) Cross-sectional SEM image of the embedded air-gap PhC LED. 


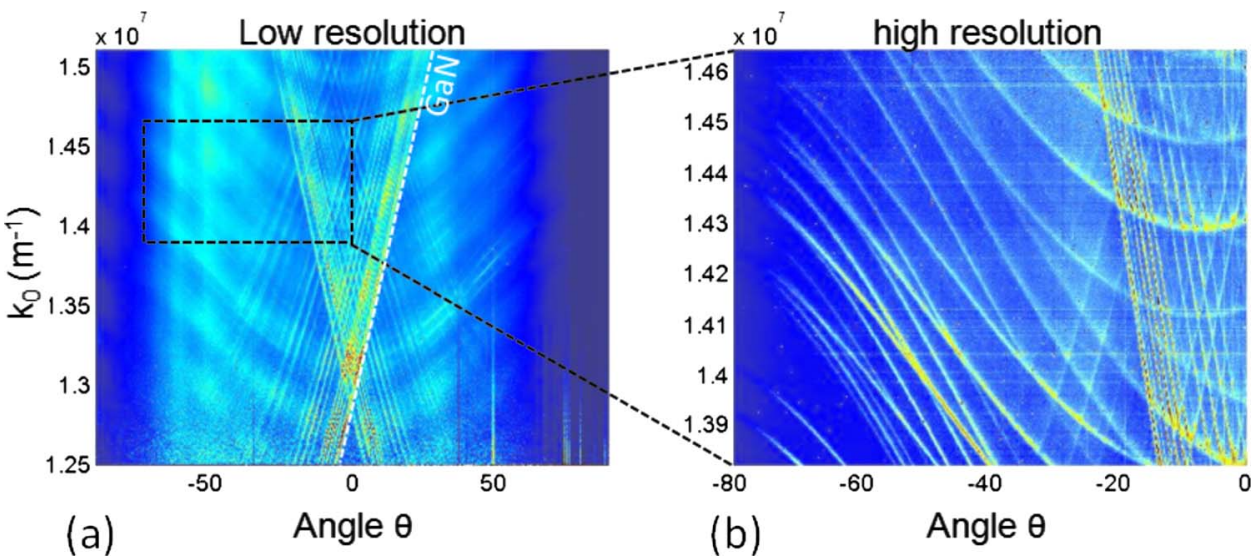

FIG. 2. (Color online) (a) Low resolution angle-resolved EL measurement, where $k_{0}=2 \pi / \lambda$, showing the diffraction of the guided modes by the embedded PhCs. (b) High-resolution angle-resolved measurement revealing the presence of many more diffracted modes.

The critical vertical dimensions of this structure, such as thickness of the cap-layer and embedded air-gap PhCs, were theoretically optimized by calculating the extraction length $\left(L_{\text {extraction }}\right)$ of the CLM, which corresponds to the distance traveled by the mode after which the fraction $\left(1-e^{-1}\right)$ of its energy is diffracted to air by the PhCs. The modal extraction length is related to the imaginary component of the wave vector $k^{\prime \prime}$ of the guided mode (or more precisely, Bloch mode) supported by the structure as $L_{\text {extraction }}=1 / 2 k^{\prime \prime}$, where $k^{\prime \prime}$ is due only to the $\mathrm{PhC}$ diffraction of this guided mode. The imaginary component of the CLM wave vector was calculated based on a full three-dimensional scattering matrix formalism ${ }^{2,10}$ considering the $\mathrm{PhCs}$ with $35 \%$ fill factor (defined as the fraction of the $\mathrm{PhC}$ surface occupied by air holes) above a $4 \mu \mathrm{m}$-thick $\mathrm{GaN}$ layer and wavelength $\lambda=460 \mathrm{~nm}$. The dependence of the calculated CLM extraction length on the cap layer and $\mathrm{PhC}$ thicknesses is shown on Fig. 1(b).

The shortest extraction length of the CLM is achieved for the thinnest possible cap layer and a relatively thin $\mathrm{PhC}$ layer $(\sim 100 \mathrm{~nm})$, which produces a weaker confinement of the CLM, resulting in a higher interaction with the embedded PhCs. The discontinuities at the dashed lines shown in Fig. 1(b) are due to the change on the mode order of the CLM as the thickness of the cavity varies and the oscillations represent variations in the vertical resonances of the Bloch mode with the $\mathrm{PhC}$ layer. ${ }^{11}$ The optimal $\mathrm{PhC}$ fill factor for a 540 nm-thick cap layer and $100 \mathrm{~nm}$-thick embedded $\mathrm{PhC}$ layer was found to be around $14 \%$. Both low fill factor and thin embedded PhCs result in weak confinement of modes in the cap layer (or equivalently, in the bottom GaN layer below the $\mathrm{PhCs}$ ). Therefore, the modes spatially extend across the $\mathrm{PhC}$ layer, which increases their diffraction strength resulting in shorter extraction lengths.

These optimal values guided the fabrication of the structure. The GaN-based embedded PhC LED was grown by metalorganic chemical vapor deposition on a sapphire substrate. The LED structure consists of a $\sim 3 \mu \mathrm{m}$-thick n-GaN followed by a $100 \mathrm{~nm}$-thick layer of air-gap PhCs, with $230 \mathrm{~nm}$ period, and $540 \mathrm{~nm}$-thick active region comprised of a $300 \mathrm{~nm}$-thick n-GaN layer, 6x-InGaN QWs emitting at $\lambda=445 \mathrm{~nm}$ and a $110 \mathrm{~nm}$-thick p-GaN layer [Fig. 1(c)]. The 2D triangular-lattice air-gap PhCs were embedded within the GaN layer by the growth method described in Ref. 12 with a $\sim 12 \%$ fill factor. The device size was $800 \times 800 \mu \mathrm{m}^{2}$ and the current was injected through a circular p-contact (diameter of $100 \mu \mathrm{m})$ in the center of the device to separate the metal absorption from the $\mathrm{PhC}$ extraction of the guided modes. Although this choice of contact scheme offers poor current spreading in the $\mathrm{p}-\mathrm{GaN}$ layer, it is optically equivalent to a perfectly reflecting metal deposited over the entire surface in flip-chip LEDs.

The measurement of the extracted guided modes was performed by an angle-resolved electroluminescence (EL) setup where the spectrum of the LED under electric bias was measured for all angles $\theta$ from $0^{\circ}$ to $180^{\circ}$. The collection of all the spectra versus angle, along the $\Gamma \mathrm{K}$ direction of symmetry of the $\mathrm{PhC},{ }^{13}$ reveals the dispersion relation of the extracted guided modes represented by the sharp lines in Fig. 2 (a), where $k_{0}=2 \pi / \lambda$. The comparison of the high-resolution angular spectra of the LEDs with embedded PhCs [Fig. 2(b)] to the one with surface $\mathrm{PhCs},{ }^{13}$ under similar conditions, reveals a higher number of modes diffracted by the embedded PhCs. In particular, well diffracted modes were observed between $-80^{\circ}$ and $-20^{\circ}$ [Fig. 2(b)] which were not observed from the surface PhC LEDs.

The extraction length of the low order modes, determined from the linewidth of the high-resolution angular spectra $^{13}$ [Fig. 2(b)], was in the range of $64-80 \mu \mathrm{m}$ which is lower than the extraction lengths of the same modes in a similar structure with surface PhCs. ${ }^{13}$ Additionally, the extraction of all low order modes was observed, in contrast to the surface PhCs. $2,3,13$

The PhC period $a=230 \mathrm{~nm}$ was designed to enhance the LED vertical emission, which can be seen from the angleresolved measurements of Fig. 2(a), where the diffracted modes are mostly concentrated at angles between $-30^{\circ}$ and $30^{\circ}$. The vertically emitted power of the LEDs as a function of the current was measured by placing a power meter $5 \mathrm{~mm}$ above the device, with a full collection angle of $53^{\circ}$ and the result is shown in Fig. 3(a). The red curves correspond to the non-PhC LEDs and the blue to the embedded PhC LEDs. The dotted curves represent the extreme performances obtained among all measured devices. Average performing devices [solid lines on Fig. 3(a)] were used for comparison, to exclude the effect of the internal quantum efficiencies (IQE) variation over the wafer, showing a 3.5-fold enhancement in the vertical output power by the embedded PhCs. This is a rather qualitative result because the present contact scheme blocks most of the direct emitted light from the non-PhC LEDs, therefore the top emitted output power cannot be fairly compared between these devices. However, it demonstrates the $\mathrm{PhC}$ ability to vertically extract the guided modes. The I-V characteristics were not modified by the inclusion of 


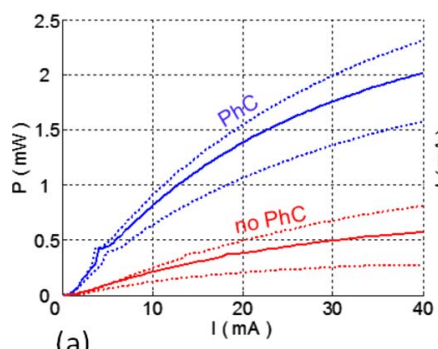

(a)

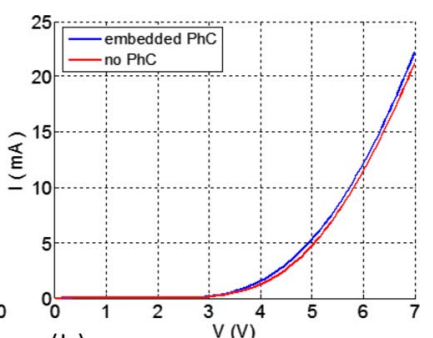

(b)

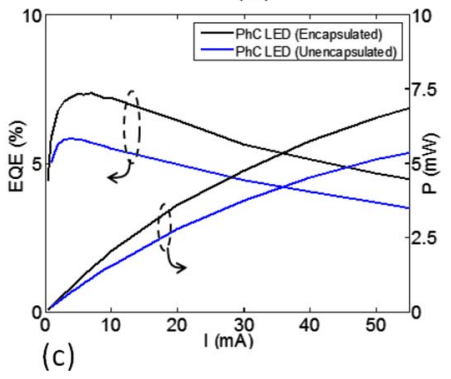

FIG. 3. (Color online) (a) Vertically emitted output power versus current for the embedded PhC LEDs and non-PhC LED. The dotted curves represent the extreme behaving and the solid line the average behaving devices. (b) $\mathrm{I}-\mathrm{V}$ characteristics of the embedded $\mathrm{PhC}$ and non-PhC LEDs. (c) EQE and total output power vs current of the unencapsulated embedded $\mathrm{PhC}$ and encapsulated embedded PhC LEDs.

the embedded PhCs. Hence no additional series resistance was introduced into the device [Fig. 3(b)].

An important figure of merit to quantify the total light extraction in the LED is the extraction efficiency, defined as $\eta_{\text {extraction }}=\mathrm{EQE} / \mathrm{IQE}$, where $\mathrm{EQE}$ and IQE are, respectively, the external and internal quantum efficiencies. The EQE as a function of current density was determined from the measurement of the total output power of the average-performing unencapsulated embedded PhC LED, using an integrating sphere [blue curve in Fig. 3(c)]. The total output power of the same devices after encapsulation with a silicone dome is shown by the black line in Fig. 3(c).

The peak IQE of these devices LEDs was estimated using a room temperature (RT) EL method. The output power was measured from a well defined surface of a single facet of the LED, where the extraction efficiency $\left(\eta_{\text {extraction }_{f}}\right)$ of the non-PhC LED is well known theoretically, ${ }^{14}$ and the rest of the chip was covered with light absorbing material. Non-PhC LEDs from the same wafer with similar output power were processed specifically for the IQE measurement, using the same processing method described in Ref. 14. The IQE was determined as $\mathrm{IQE}_{\text {peak }}=\mathrm{EQE}_{\text {peak }} / \eta_{\text {extraction }_{f}}$ resulting in $7.9 \%$ for the non-PhC LED. The $\eta_{\text {extraction }}$ of the unencapsulated embedded PhC LED was then estimated to be 73\% assuming that the IQE does not vary considerably with the introduction of embedded PhCs in the structure. Although the embedded $\mathrm{PhC}$ efficiently extracts all guided modes from the GaN slab, it does not extract the modes guided in the sapphire substrate (corresponding to $\sim 22 \%$ of the emitted energy from the QWs (Ref. 7) plus some guided modes diffracted from GaN to sapphire by the PhCs). To increase the extraction of the sapphire modes to air, the same embedded PhC LED was encapsulated with a silicone dome yielding an extraction efficiency of $94 \%$.

The IQE was also estimated by the widely used temperature-dependent photoluminescence (PL) method,

where the IQE at RT is determined from the ratio of output powers at RT and low temperature (LT), assuming the IQE at LT $(4 \mathrm{~K})$ is $100 \%$. However, we expect that our materials may contain a rather high density of non radiative centers which would not be completely suppressed at LT. Therefore use of this method allows us to only set an upper bound on the IQE; $12.6 \%$ for the embedded PhC LEDs, and $15.5 \%$ for non-PhC LEDs. The estimated lower bound for $\eta_{\text {extraction }}$ of the unencapsulated devices was then $46 \%$ and $59 \%$ for the encapsulated embedded PhC LED.

The high extraction efficiency obtained from the encapsulated embedded PhC LEDs is in agreement with the very good extraction of the guided modes in both $\mathrm{GaN}$ and sapphire slabs by the embedded PhCs, however the IQE estimation from both methods needs to be carefully considered. While the RT EL method is a more reliable technique, it was measured from different devices (from the same wafer with similar output power), due to the different processing method required. On the other hand, the temperature dependent PL method, realized on the same devices used in the EQE measurement, overestimates the IQE and assumes similar carrier injection in QWs from PL and EL.

In conclusion, the embedded PhC LEDs reported in this letter were designed to yield short extraction length of the guided modes, improving the competition between $\mathrm{PhC}$ assisted light extraction and dissipation mechanisms. The diffraction of all low order modes was observed in contrast to surface PhC LEDs. High extraction efficiency devices, close to unity, were demonstrated with directional light emission, which makes the embedded air-gap PhCs an excellent candidate for high efficiency and high brightness LEDs.

This work was supported by DOE under Project No. DE-FC26-06NT42857 and DOE-EFRC under Project No. DE-SC0001009.

${ }^{1}$ J. J. Wierer, M. R. Krames, J. E. Epler, N. F. Gardner, M. G. Craford, J. R. Wendt, J. A. Simmons, and M. M. Sigalas, Appl. Phys. Lett. 84, 3885 (2004).

${ }^{2}$ A. David, C. Meier, R. Sharma, F. S. Diana, S. P. DenBaars, E. Hu, S. Nakamura, C. Weisbuch, and H. Benisty, Appl. Phys. Lett. 87, 101107 (2005).

${ }^{3}$ K. McGroddy, A. David, E. Matioli, M. Iza, S. Nakamura, S. DenBaars, J. S. Speck, C. Weisbuch, and E. L. Hu, Appl. Phys. Lett. 93, 103502 (2008).

${ }^{4}$ J. J. Wierer, A. David, and M. M. Megens, Nat. Photonics 3, 163 (2009).

${ }^{5}$ C.-F. Lai, C.-H. Chao, H.-C. Kuo, H.-H. Yen, C.-E. Lee, and W.-Y. Yeh, Appl. Phys. Lett. 94, 123106 (2009).

${ }^{6}$ M. R. Krames, O. B. Shchekin, R. Mueller-Mach, G. Mueller, L. Zhou, G. Harbers, and M. G. Craford, J. Disp. Technol. 3, 160 (2007).

${ }^{7}$ A. David, T. Fujii, R. Sharma, K. McGroddy, S. Nakamura, S. P. DenBaars, E. L. Hu, C. Weisbuch, and H. Benisty, Appl. Phys. Lett. 88, 061124 (2006).

${ }^{8}$ E. Matioli, M. Iza, Y.-S. Choi, F. Wu, S. Keller, H. Masui, E. Hu, J. Speck, and C. Weisbuch, Phys. Status Solidi C 6, S675 (2009).

${ }^{9}$ A. David, B. Moran, K. McGroddy, E. Matioli, E. L. Hu, S. P. DenBaars, S. Nakamura, and C. Weisbuch, Appl. Phys. Lett. 92, 113514 (2008).

${ }^{10}$ S. G. Tikhodeev, A. L. Yablonskii, E. A. Muljarov, N. A. Gippius, and T. Ishihara, Phys. Rev. B 66, 045102, 2002.

${ }^{11}$ A. David, H. Benisty, and C. Weisbuch, J. Disp. Technol. 3, 133 (2007).

${ }^{12}$ E. Matioli, S. Keller, F. Wu, Y.-S. Choi, E. Hu, J. Speck, and C. Weisbuch, J. Appl. Phys. 106, 024309 (2009).

${ }^{13}$ E. Matioli, B. Fleury, E. Rangel, E. Hu, J. S. Speck, and C. Weisbuch (unpublished).

${ }^{14}$ A. Getty, E. Matioli, M. Iza, C. Weisbuch, and J. S. Speck, Appl. Phys. Lett. 94, 181102 (2009). 